Techniques \& Culture

Revue semestrielle d'anthropologie des techniques

$56 \mid 2011$

Habiter le temporaire

\title{
Des-Équilibres humains
}

Une introduction

Human Imbalances. An introduction

Agnès Jeanjean

\section{(2) OpenEdition}

Journals

Édition électronique

URL : https://journals.openedition.org/tc/5262

DOI : $10.4000 /$ tc. 5262

ISBN : 1952-420X

ISSN : 1952-420X

Éditeur

Éditions de l'EHESS

Édition imprimée

Date de publication : 5 septembre 2011

Pagination : 16-29

ISBN : 978-2-7351-1410-8

ISSN : 0248-6016

Référence électronique

Agnès Jeanjean, « Des-Équilibres humains », Techniques \& Culture [En ligne], 56 | 2011, mis en ligne le 30 novembre 2011, consulté le 29 septembre 2022. URL : http://journals.openedition.org/tc/5262 ; DOI : https://doi.org/10.4000/tc.5262 


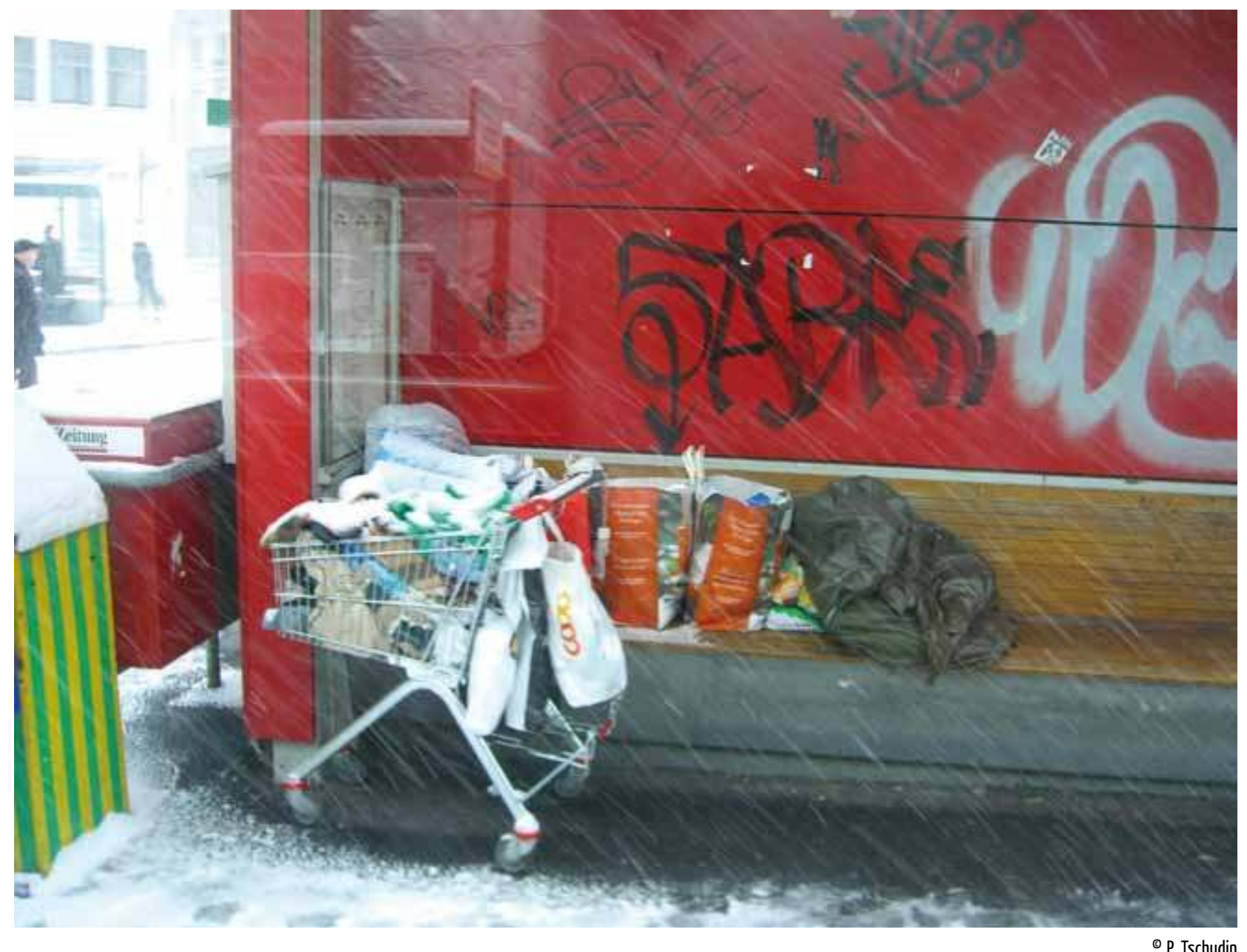




\section{DES-ÉQUILIBRES HUMAINS}

\section{Une introduction à « Habiter le temporaire »}

En France et plus généralement dans les pays riches, nous assistons depuis quelques années à une recrudescence de formes diverses d'habitats posés, temporaires et mobiles. Les habitations de fortune auto-construites sont omniprésentes dans les paysages ruraux et urbains ${ }^{1}$. Leur présence est pleinement articulée à la fragilisation économique et sociale des populations, aux dispositifs institutionnels d'organisation et de gestion des territoires y compris au niveau mondial (Bauman 2004 ; Agier 2002), de même qu'à la flexibilité exigée par les modes de production et les choix économiques dominants. Comme en attestent de nombreux travaux d'historiens mais aussi d'archéologues, repris dans ce numéro par Ingrid Sénépart, la mobilité et l'itinérance des travailleurs, le nomadisme des ouvriers et l'errance des populations ne sont pas des phénomènes nouveaux. Ils ne sont par ailleurs pas systématiquement synonymes de précarité sociale. Dans cette livraison de Techniques \& Culture, les contributions d'Alain Beeching, d'Hélène Claudot-Hawad, de Laurent Dousset, de Marc Vincent et Lucie Dupré sont là pour nous le rappeler. Les bidonvilles sont par ailleurs nombreux de par le monde et ils étaient encore bien présents dans le paysage parisien jusque dans les années 1970, comme le souligne Colette Pétonnet dans ce Thema. Toutefois, nous pouvons envisager les phénomènes récents en les reliant aussi au contexte contemporain. Cette proposition sera pour nous l'occasion de convoquer des auteurs que nous n'avons pu accueillir et dont nous regrettons l'absence. Si, à l'instar d'Alain Birh (2007), nous nous tournons vers les écrits de Marx et Engels (1847), le chômage, la précarité et l'insécurité peuvent être pensés comme des phénomènes socio-économiques constitutifs du salariat dont seules changeraient les formes historiques ${ }^{2}$. Pierre Bourdieu, quant à lui, considérait que nous nous situons 
dans un mode de domination sans précédent même s'il ressemble au « capitalisme sauvage des origines » et fondé sur «l'institution d’un état généralisé et permanent d’insécurité visant à contraindre les travailleurs à la soumission, à l'acceptation de l'exploitation ». Il proposait, pour le qualifier, d'adopter la notion de «flexploitation» (Bourdieu 2008 : 99). C'est aussi à partir d'un état historique du capitalisme : « un monde connexionniste », que Luc Boltanski et Ėve Chiapello proposent de penser les formes contemporaines d'exclusion, d'inclusion et de précarité. Ils associent ces dernières à des rapports de domination des « mobiles» sur les « immobiles » : «Dans un monde connexionniste où la grandeur suppose le déplacement, les grands tirent une partie de leur force de l'immobilité des petits » (Boltanski et Chiapello 1999 : 448).

Dans ce numéro, Béatrice Mésini et Arnaud Le Marchand montrent que les habitations légères et mobiles sont adaptées au contexte contemporain « flexible » et " connexionniste ", ainsi qu'aux formes actuelles de précarité sociale et de pauvreté. Les évolutions du prix du foncier et des crédits bancaires, la crise du logement ${ }^{3}$, les dispositifs publics mis en œuvre pour faire face à la fragilisation des individus, leurs orientations et leurs insuffisances, de même que les lois et les modalités de leurs applications, examinées ici par Chantal Iorio, ont des effets directs sur les modes d'accession au logement et sur les formes d'habiter et de précarité. Ainsi, les logis de fortune, les occupations « sans droit ni titre » peuventils être envisagés comme le résultat d'une coproduction entre un ordre social et des individus précarisés (Legros 2010) et in fine, comme un habitat dans tout ce que cette notion comprend d'interactions avec un environnement naturel et social ${ }^{4}$.

\section{Le produit d'une dialectique}

Voici maintenant trente ans que Colette Pétonnet a proposé de penser les bidonvilles parisiens des années soixante et soixante-dix comme possédant « une fonction de passage transitoire entre deux mondes " pour les immigrés récemment arrivés à Paris et économiquement fragiles (Petonnet 1979 : 42). Cela dans la mesure où le bidonville était une étape vers d'autres formes de logement inscrites de façon plus légitime dans la ville. Elle a mis en exergue les équilibres à l'œuvre, l'existence d'une organisation collective, les éléments de continuité et les liens avec l'espace urbain environnant développés par cet habitat auto construit (Pétonnet 1979, 1982). C'est aussi ce que montrent les récents travaux d'Olivier Legros (Legros 2010) consacrés aux bidonvilles et aux « villages d'insertion » d'Aubervilliers, de Saint-Denis et de Saint-Ouen. Ce constat vaut pour d'autres types d'abris auto-construits, y compris en zone rurale et en des temps plus éloignés. C'est le cas notamment des cabanes de charbonniers du sud de la France aux xix ${ }^{e}$ et xx ${ }^{e}$ siècles étudiées par Ada Acovitsiòti-Hameau et Philippe Hameau (1996). Ces cabanes, établies en forêt sur les lieux de production du charbon, avaient une double fonction d'habitation et de surveillance du processus de combustion. Ada Acovitsiòti-Hameau montre bien que lorsque le charbonnage était le fait de migrants, la cabane jouait un rôle central dans le processus d'intégration sociale au sein d'une région ou d'un village :

« Les forestiers ne sont ni ancrés, ni bloqués dans leur forêt, mais ils développent des stratégies diverses pour approcher et s'allier la société qui pourrait les accueillir. 
La régularité des déplacements au sein d'une micro-région définie, aboutit à l’acquisition de droits d'autochtonie sur cette région, tant par la connaissance intime des lieux qu'au travers des pied-à-terre concrets et personnels (« les cabanes ») édifiés dans son périmètre » (Acovitsiòti-Hameau 2000 : 119).

Les opérations de destruction de bidonvilles, d'expulsion, voire de relogement, lorsqu'elles rompent trop brutalement ces équilibres, empêchent ce mouvement classique d'insertion sur un territoire. Elles engendrent un surcroît de misère et une précarité accentuée des habitants. C'est ainsi qu'observant les effets de la destruction des bidonvilles parisiens et du relogement des bidonvillois en cité de transit, Colette Pétonnet (1979) y a vu la production de victimes émissaires. Les habitants, par le biais de relogements aveugles aux réseaux de connaissances, aux liens familiaux et aux articulations déployées entre le bidonville et le reste de la ville, ont perdu la possibilité de maîtriser leur habitat et de ce fait leur inscription au sein d'un territoire et d'une société. C'est bien ici cette place au milieu des autres, cette fragile mais effective expression de l'« habiter » et les formes qu'elle revêt, que les mesures de destruction, de relogement et d'expulsion condamnent. Elles œuvrent à la façon dont Lévi-Strauss dans un passage très célèbre de Tristes tropiques décrit l'action des missionnaires salésiens de la région du Rio das Garças :

« (...) le plus sûr moyen de convertir les Bororo consiste à leur faire abandonner leur village pour un autre où les maisons sont disposées en rangées parallèles. Désorientés par rapport aux points cardinaux, privés du plan qui fournit un argument à leur savoir, les indigènes perdent rapidement le sens des traditions, comme si leurs systèmes social et religieux (...) étaient trop compliqués pour se passer du schéma rendu patent sur le plan du village et dont leurs gestes quotidiens rafraîchissent perpétuellement les contours » (Levi-Strauss $1995: 255)^{5}$.

Ces opérations donnent clairement à observer combien la précarité - lorsqu'elle n'est pas entendue au sens ontologique mais plutôt comme une forme d'existence menacée, soumise à un pouvoir extérieur à elle et dont elle dépend - est le produit de dispositifs sociaux (Le Blanc 2007). Les formes qu'elle revêt et les individus concernés, diffèrent selon les actions engagées ou non par les politiques publiques. Il peut s'agir par exemple de régularisations des occupations « sans droit ni titre », d'expulsions pures et simples, d'opérations de relogement accompagnées du déplacement des individus et de la destruction de leurs habitations. Par ailleurs les pouvoirs publics peuvent choisir de prendre en charge les problèmes liés à la pauvreté. Il peut arriver également qu'ils en délèguent la « gestion » aux acteurs du marché ${ }^{6}$. De nombreux auteurs ont analysé ces différents dispositifs et leurs effets, notamment en ce qui concerne les bidonvilles tout autour du monde. Ce mécanisme de précarisation est visible au travers des mesures prises vis-à-vis des habitations mobiles et qui déterminent les possibilités ou impossibilités de stationner, de même que des durées autorisées, rendant plus ou moins précaire et temporaire l'insertion des habitations et de leurs habitants sur un territoire. On sait depuis maintenant longtemps, et notamment grâce aux travaux de Maurice Halbwachs et de Roger Bastide, l'importance que revêt pour les individus et les groupes, l'inscription de leurs univers de représentation dans l'espace. La condition d'existence de repères temporels et de formes de continuité repose sur leur matérialisation. Ici apparaît clairement le caractère éminemment politique de la gestion des espaces mais aussi des habitations, 
de l'acte de construire et de celui d'habiter ${ }^{7}$. Bien évidemment ces actes sont d'autant plus critiques qu'ils s'inscrivent dans des marges ${ }^{8}$ et des espaces interstitiels, dont on sait les dimensions symboliques mais aussi créatrices et « ouvertes » (Trasher 1927 ; Remy 1996) et qu'ils se déploient sur des terrains non constructibles, selon des règles non conformes aux codes de l'urbanisme, au respect de la propriété privée, ainsi qu'aux normes en matière de circulation des hommes et des biens. Rappelons au passage que la pensée nomade est envisagée par certains philosophes (Nietzsche, Deleuze et Guattari) comme une puissante forme critique (Deleuze \& Guattari 1980).

\section{Injonctions contradictoires : des rapports de norme}

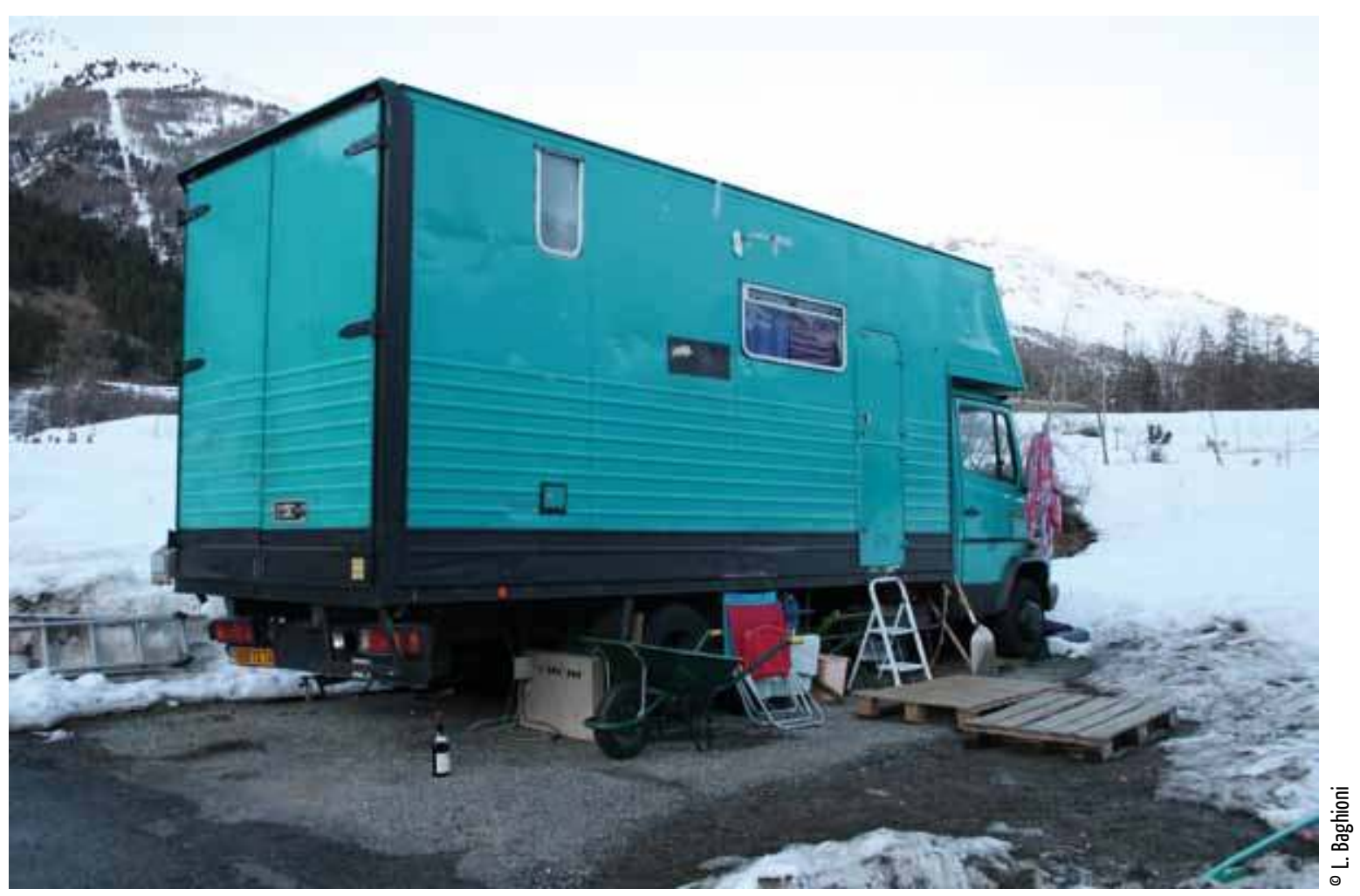

Camion aménagé par un couple de saisonniers du tourisme, installé à proximité de la station de Serre Chevalier (05), février 2009.
Comme le relève Colette Pétonnet, dans ce volume, les habitations qui font l'objet de ce Thema sont environnées d'injonctions contradictoires multiples et donnent à voir de nombreux paradoxes. Ceux-ci soulèvent d'importantes questions sociétales. Nous avons évoqué le traitement social des bidonvilles, les démolitions, les expulsions et la précarité que génère leur éventualité. Il en va de même à propos d'autres expressions de l'habiter « sans droit ni titre » déployées par les individus et les groupes précarisés 
et sur lesquelles s'exercent des interdictions, des mesures ou des menaces de démolition, d'expulsion. Nous pensons aux squats décrits par Florence Bouillon (Bouillon 2009), aux séjours permanents en terrain de camping étudiés par Nicolas Bernard (Bernard 2006), aux installations de mobile-homes sur des terrains privés (Poulain 2005). Il en va de même des phénomènes dits de « cabanisation » qui conduisent à occuper de façon permanente, en les transformant, des abris construits sans permis et destinés à l'origine à une occupation temporaire fondée sur des activités de travail ou de loisir (Raveneau et Sirost 2011). Les habitations mobiles et leurs habitants, sont eux aussi face à des contradictions. C'est le cas des travailleurs saisonniers qui vivent dans des camions et ne trouvent pas de lieu où stationner à proximité des villes ou des villages au sein desquels ils déploient leur activité saisonnière. Cet habitat semble pourtant assez bien adapté à leur mobilité professionnelle, de même qu'à leurs revenus irréguliers, et aux tarifs prohibitifs des locations touristiques (Baghioni 2007). Les gens dits « du voyage » sont, quant à eux, soumis aux injonctions contradictoires de devoir circuler sans pouvoir stationner (Aubin 1996 ; Charlemagne 1996), de trouver impérativement une place dans des « aires d'accueil » ou des « terrains désignés », saturés (Bordigoni dans ce numéro), ou encore de se sédentariser sans avoir le droit ni les moyens de rester au même endroit plus de trois mois d'affilée. Ainsi, lorsqu'ils n'ont pas la possibilité d'acquérir un terrain constructible, trouvent-ils des solutions précaires en tournant sur des terrains privés et des aires d'accueil situés sur le territoire dont ils se pensent être les habitants. Pour rester sur place, ils sont finalement poussés à être mobiles.

La valorisation de l'accès à la propriété, telle qu'elle a lieu en France par exemple où le pouvoir politique central promet une «France de petits propriétaires » et élabore des projets de loi facilitant et accélérant les procédures de démolition des habitats mobiles, posés et construits « sans droit ni titre $»^{9}$ soulève également des paradoxes. Ici s'exprime clairement un rapport de norme, celui-ci oppose des habitations fixes, susceptibles de traverser le temps, voire de constituer un patrimoine économique transmissible, et situées sur des zones déclarées constructibles à d'autres qui sont mobiles, éphémères, fragiles et inscrites de façon plus floue dans le temps et dans l'espace. Laccession à la propriété et à l'habitat en dur sont envisagés comme des opérations fiables, garantissant leurs habitants contre un rapport incertain à l'avenir. Or, les dernières crises immobilières étasuniennes et espagnoles viennent de démontrer que l'accession à la propriété pouvait devenir un facteur aggravant de précarité. Il est par ailleurs autour de nous de nombreux exemples d'habitations en dur à la durée de vie assez brève, nous pensons en particulier aux destructions de barres d'immeubles sociaux (Calame 1987). La fixité et la solidité des habitations sont en outre envisagées comme inscrites dans une longue tradition. Dans un article déjà ancien, François Calame, prenant pour exemple l'habitat picard pré-industriel remet en question l'évidence d'une telle tradition. Il décrit un habitat constitué de matériaux en apparence fragiles (bois et torchis) pouvant traverser le temps mais également donner lieu à des réemplois. Il signale par ailleurs, dans le nord-ouest de l'Oise et le sud-ouest de la Somme, de nombreuses opérations de transfert de bâtiments : « les bâtiments, qu'ils soient grange ou logement, peuvent être démontés, stockés en pièces détachées, transportés, remontés. Ils peuvent être également roulés sur des distances de plusieurs centaines de mètres » (Calame 1987 : 85). Cet habitat «traditionnel », considéré comme un bien meuble, bouge. Il en va de même des vagi dont Marc Bordigoni, s'appuyant sur un corpus photographique exceptionnel, examine - dans la partie Curiosa de la revue - la construction, les usages et la place dans l'espace physique et social de la France des années soixante. 
Les paradoxes que nous venons de souligner nous conduisent à insister sur les rapports de norme à l'œuvre autour des modes d'habiter développés par des populations pauvres et précarisées et plus particulièrement des habitations auto-construites, temporaires et mobiles.

\section{Des pratiques subversives}

Dans un ouvrage collectif consacré aux cabanes, Bernard Picon souligne la dimension utopique de ces dernières:

« Les cabanes ignorent les catégories juridiques du bâti et du non bâti, du dedans et du dehors, du naturel et de l'artificiel. Étant tout à la fois, elles échappent aux grandes juridictions habilitées à légiférer sur les territoires urbains, ruraux et naturels ; elles constituent sur leurs marges des refuges contre ces machines à écarteler. Elles relèvent de l'insupportable univers du flou » (Picon 2000 : 332).

Il évoque les cabanes de loisir liées à des activités secondaires. C'est le cas de celles de Beauduc examinées ici par Laurence Nicolas, ou encore de la pratique du camping (Sirost dans ce volume). Toutes les habitations présentées dans cette introduction et dans le Thema ne sont pas forcément secondaires. Elles possèdent toutefois une puissance critique. Certaines, de par leur existence même, manifestent la présence au sein de l'ordre social, d'une «part maudite », d'un reste irréductible: on ne peut pas interdire une pratique lorsque celle-ci est indispensable. Elles sont liées par ailleurs à des activités de construction et de survie, à des modes de vie subversifs dès lors qu'ils se situent dans les marges de l'économie marchande. Lorsqu'ils sont auto-construits ou de fortune, les habitations ou abris s'appuient sur l'utilisation de restes, ou de déchets, de matériaux tirés du sol. Ces assemblages d'objets relevant d'univers d'ordinaire disjoints dans l'espace ou dans le temps, sont le produit de pratiques de détournements. Leur édification mobilise des savoirs et savoir-faire hétérogènes (naturalistes, professionnels, juridiques, ...). Ils engendrent des effets de sens, perturbent les catégories établies. Comme les pratiques secondaires observées par Erving Goffman au sein d'institutions psychiatriques (Goffman 1984), ils relèvent de mécanismes infiniment et irréductiblement humains. Ils mettent en exergue la puissance critique et subjectivante des objets et des matières y compris ceux en apparence les plus insignifiants. Cette dernière dimension est particulièrement présente dans les contributions de Laurence Nicolas et de Sylvain Brino. Arnaud le Marchand enrichit cette perspective dans la mesure où il s'intéresse à des habitations marginales (les conteneurs) rattrapées par les normes, la société générale et les modes de production dominants. C'est ici la question de la normalisation qui est soulevée et de ce qui advient de la dimension subversive et de la singularité dès lors qu'elles sont englobées dans un discours politique, urbanistique, esthétique, conservatoire, légitime et légitimant. 


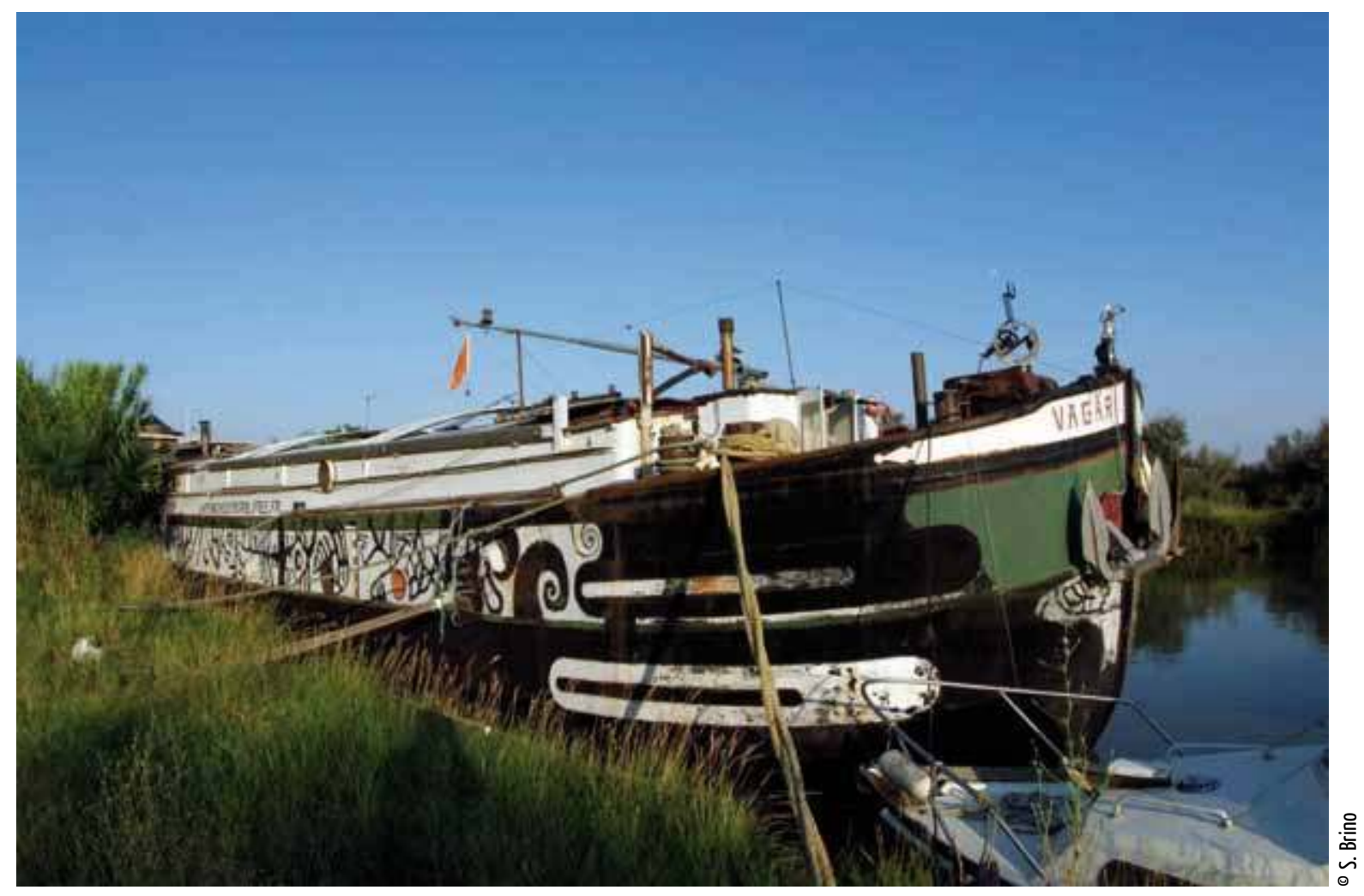

Péniche exposition / atelier Vagari, juillet 2011

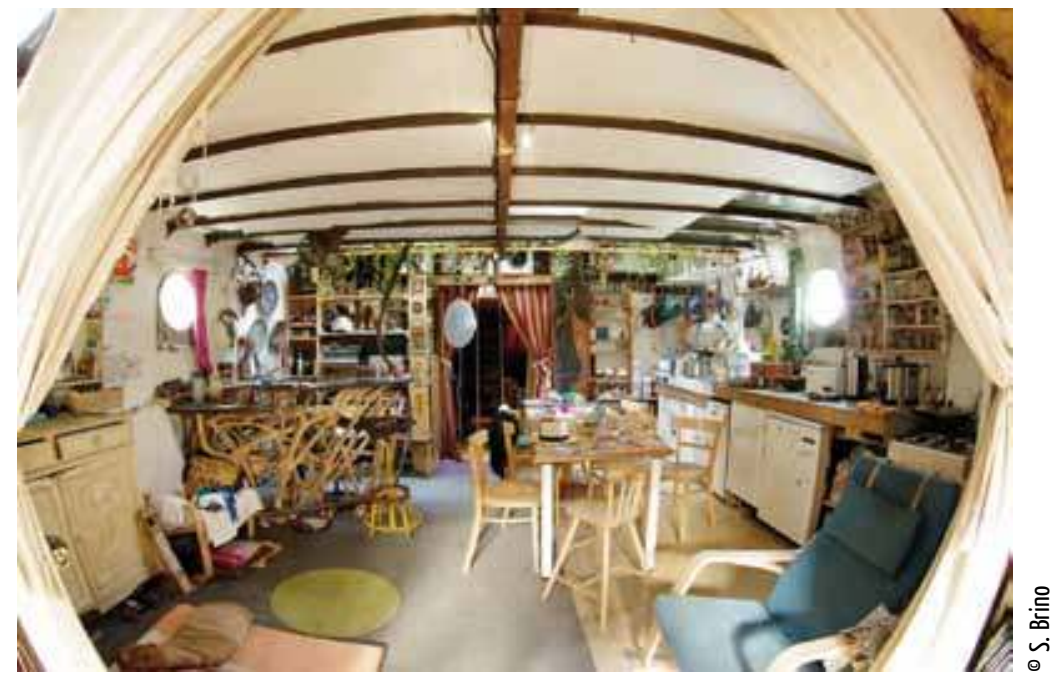

Ancienne péniche de transport, gabarit Freycinet (38 m x $5 \mathrm{~m}$ ), reconvertie par Pierre Besson plasticien polyvavent, en atelier de sculpture, salle d'exposition, habitation. Elle est basée depuis plusieurs années à la halte nautique de Galician (Gard). 


\section{Confins et déplacements}

Les habitations et abris de fortune bousculent et déplacent les limites, spatiales mais aussi humaines. Ils déploient une palette infinie de combinaisons lesquelles vont jusque dans les confins de la misère humaine et du dénuement. Des textes contemporains explorent cette graduation. Nous pensons ici aux travaux de Pascale Pichon et plus particulièrement à son article «Vivre sans domicile fixe : l'épreuve de l'habitat précaire ». Dans ce texte, il n'est déjà plus question d'habitations mais d'abris et l'auteure distingue les abris « sauvages » d'abris plus «sûrs ». Au fil de rencontres et d'événements heureux ou malheureux, les habitants passent d'un abri à un autre.

\section{SDF cartonniers sous un pont de la rivière Kamogawa, Kyoto, 2009}

On remarquera l'habitat à deux étages, l'un en haut avec le matériel et les instruments de l'activité de récupération en contact avec les piétons très nombreux en ce lieu, et en bas, l'espace de vie. Comme dans l'habitat traditionnel en maison, le nécessaire au couchage est enfermé dans un coffre et sorti pour la nuit.
«Le squat mais aussi quelques autres abris, tels que ou la cabane de jardin ou le wagon désaffecté par exemple, apportent une sécurité, une stabilité et un confort qui, quoique relatifs, s'opposent à l'occupation éphémère et risquée de l'abri sauvage, celui du renfoncement de porche, de la cachette dans le jardin public, ou du sous-sol de parking... seul celui qui occupe un abri suffisamment sûr la nuit préserve le maintien de soi » (Pichon 2002: 17).

Patrick Gaboriau s'interroge à propos de la façon dont les clochards disent ces abris, et reprenant les mots de ses informateurs évoque des « coins» (Gaboriau 1993 : 121).

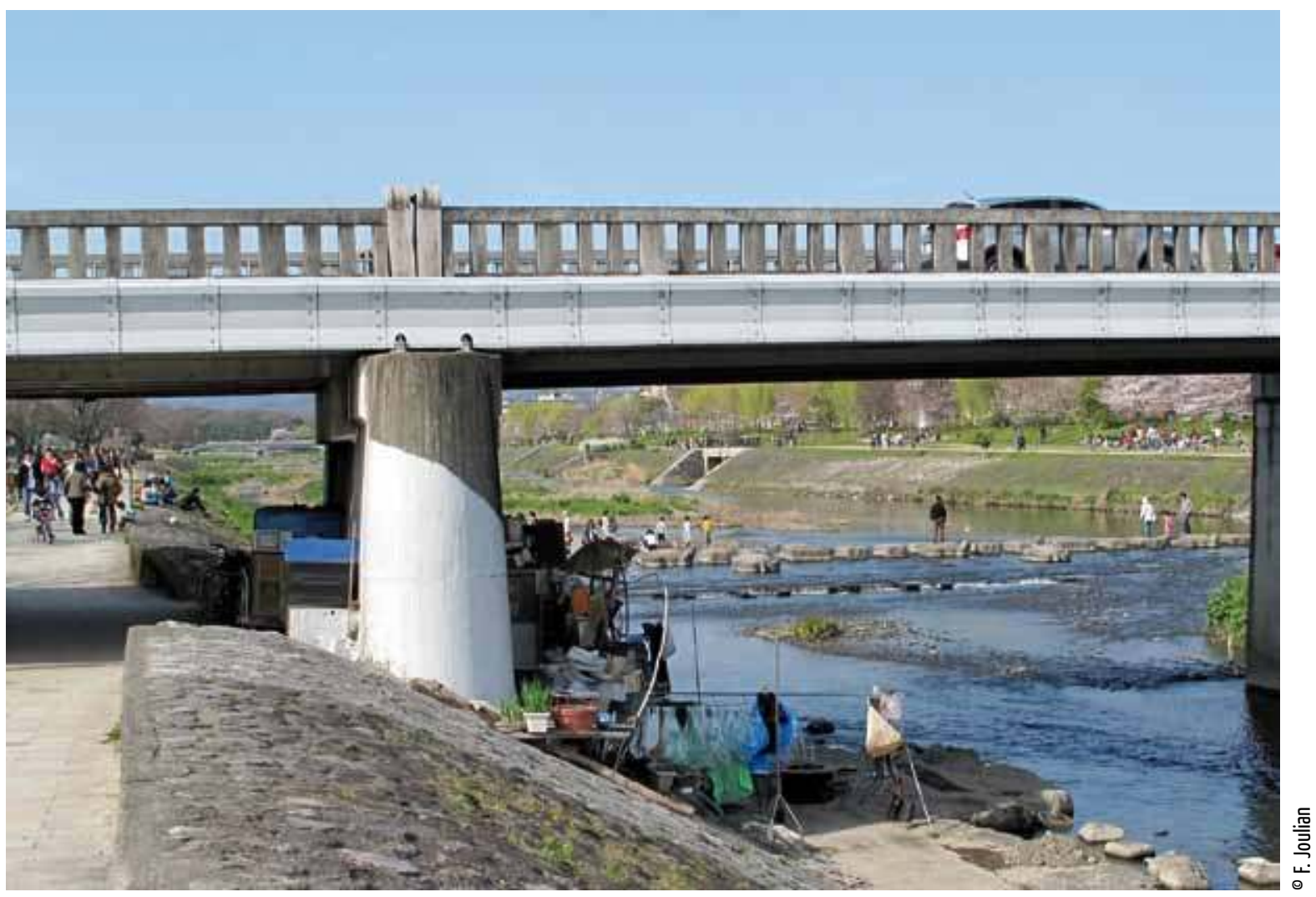


Un autre texte auquel nous pensons est celui d'Evelyne Desbois à propos des abris de fortune construits, aménagés et occupés sur le front par les soldats français lors de la guerre de 14-18 (Desbois 2002):

« Ici encore la diversité frappe. Les abris se succèdent sans se ressembler en fonction des mouvements du front, des avancées ou des retraites. Il peut s'agir de cagnas construites en plusieurs semaines et occupées de façon sédentaire, ou d'abris disposés le long d'un parcours plus ou moins glorieux et occupés le temps d'une nuit. La liste des abris est hétéroclite : granges, trottoirs, fossés, greniers, champignonnières, pavillons cossus ou « folies » récemment quittés par leurs occupants devant l'avancée des troupes ennemies, tranchée, abris de rondins, corps des compagnons de route, ...».

\section{Mouvements d'un logis ... à l'autre}

Ces derniers exemples sont l'occasion de revenir sur une dimension importante qui concerne les déplacements d'un logis vers un autre, d'un lieu vers un autre. Ces mouvements, à l'instar des « figures cheminatoires » proposées par Michel de Certeau (1990), instaurent et expriment des liens de complémentarité, des relations à un centre ou bien l'absence de centre, des disjonctions, des relations réticulaires, des éclatements. Les coins ou abris sauvages peuvent par exemple revêtir des structures de complémentarité éclatées en divers lieux de l'espace urbain, opérer une dispersion des fonctionnalités (un coin pour dormir dans le hall d'un immeuble, un autre pour manger dans un autre quartier, un «salon » aménagé sur une place publique située trois rues plus loin, un lieu où se laver...). Des forces centrifuges sont à l'œuvre ainsi que d'autres, centripètes. Nous l'avons signalé plus haut à propos de la fonction de transition et d'intégration potentiellement assurée par les habitats de fortune lorsque leur occupation est temporaire. Les études archéologiques nous aident à être attentifs aux relations de complémentarité (Sénépart et Beeching dans ce Thema). L'observation de situations contemporaines montre combien cet aspect est important pour penser les habitats, leurs habitants et la place qu'ils occupent au sein des sociétés. Lorsque les mouvements sont empêchés ou à l'inverse contraints, lorsque le sens du mouvement est perdu, lorsque l'énonciation est dissipée au profit de mobilités qui n'ont d'autres formes que la dispersion ou la fuite au point d'en devenir insensées, le temporaire et le mobile deviennent alors synonymes de précaire. Il en va ainsi des Nomades Touaregs et des Aborigènes d'Australie présents dans cette livraison au travers des articles d'Hélène Claudot-Hawad et de Laurent Dousset, ou encore des pratiques de camping dont Olivier Sirost, s'appuyant sur une approche historique, met en lumière le caractère réversible.

Cette introduction porte principalement et délibérément sur la société occidentale. En ce sens elle n'est pas exactement représentative du Thema mais c'est bien ce questionnement « occidentalo-centré » qui nous a conduites à faire appel à des auteurs étudiant des situations éloignées les unes des autres dans le temps et l'espace. Nous avons souhaité rapprocher des points de vue et des situations. À la façon des agencements d'objets à la fois improbables, signifiants et subversifs photographiés par Sylvain Brino dans les cabanes de Lunel (Hérault), nous espérons que la réunion au sein d'un même tome de tous 
ces regards, issus de disciplines diverses, sur des habitats temporaires, mobiles, parfois précaires, décale et enrichit la perspective actuellement développée sur les questions de précarité et d'habitat. Lors des journées d'études qui ont précédé la préparation de ce numéro, Colette Pétonnet avait intitulé sa communication « Les équilibres humains », c'est bien cette dimension humaine que nous espérons convoquer au travers de cet assemblage. En plus des auteurs que nous regrettons de n'avoir pas pu intégrer au numéro, il est également des habitations, des abris qui auraient dû trouver leur place ici. Nous pensons aux habitations fluviales, aux yachts et autres habitats temporaires et mobiles pratiqués par les plus riches, mais la liste serait longue et les lecteurs à leur tour établiront de nouveaux rapprochements signifiants.

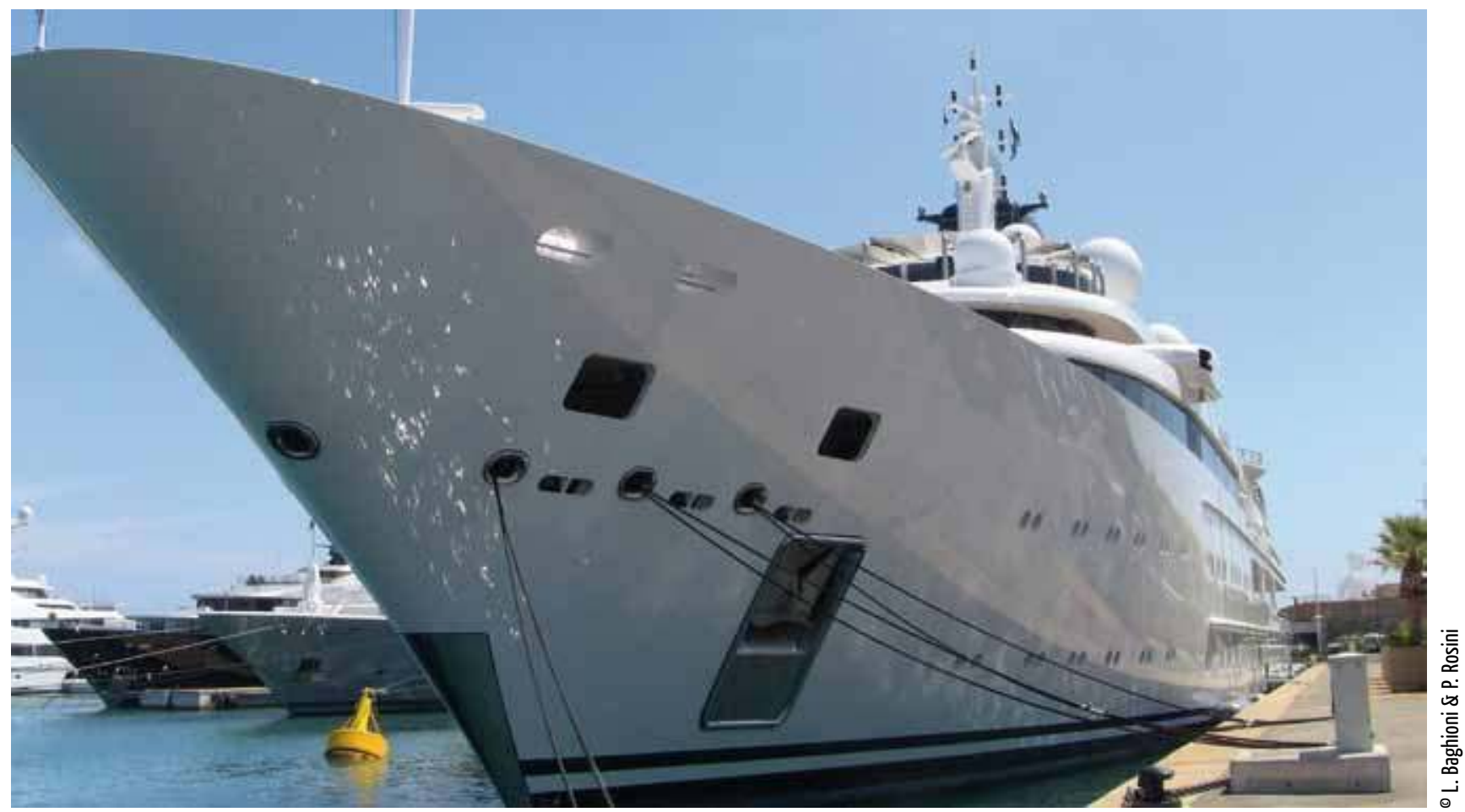

Yacht amarré au quai Camille Rayon, dit « Quai des milliardaires » Antibes, juillet 2011. 


\section{NOTES}

1. D'après la Fondation Abbé Pierre et les sources Insee, en France, en 2006, 85000 personnes vivaient dans des habitations de fortune (cabanes, constructions provisoires). 600000 autres vivaient dans la rue, à l'hôtel, au camping, dans des mobile-homes, chez des tiers ou dans des structures d'hébergement et d'insertion (Fondation Abbé Pierre, Rapport mallogement 2011 : http://www.fondation-abbe-pierre. fr/index.php).

2. «La bourgeoisie ne peut exister sans révolutionner sans cesse les instruments de production, ce qui veut dire les rapports de production, c'est-à-dire l'ensemble des rapports sociaux (...). Ce bouleversement continuel de la production, ce constant ébranlement de tout le système social, cette agitation et cette insécurité perpétuelles distinguent l'époque bourgeoise de toutes les précédentes » (K. Marx \& Engels F 1847. chapitre I)[en ligne].

3. Florence Bouillon souligne la particularité de cette « crise » qui ne relève pas forcément d'une insuffisance globale de logements mais d'une inadéquation entre l'offre et la demande (Bouillon 2009: 7).

4. Ici nous adoptons la notion d'habitat proposée par Robert Cresswell : « ...ce qui est structure du cadre bâti. Il s'agira du rapport des différents éléments construits entre eux et avec la nature. Les agglomérations, les réseaux d'occupation du sol par les constructions seront donc la forme d'habitat propre à chaque société. L'unité de base est ici évidemment le groupe » (Cresswell 2010 [1976: 41].
5. Voir aussi les « camps de regroupement» mis en place par l'administration française pendant la guerre d'Algérie.

6. Privatisation de la gestion des aires d'accueil, ou encore appel à des consortium privés pour mettre en œuvre des programmes urbains de résorption de bidonvilles (Jeanjean 2007).

7. Le conflit Israëlo Palestinien et les mouvements de colonisation des territoires occupés nous le rappellent inlassablement.

8. Dans ses travaux, Michel Agier montre combien les marges y compris dans les situations extrêmes de dépouillement de soi que sont les camps de réfugiés donnent lieu à des productions spatiales, en l'occurrence des productions urbaines qu'il qualifie toutefois d'amputées « La gestion des espaces sous le régime du gouvernement humanitaire fait des camps un espace de pure attente et sans sujet. À ce principe de pouvoir s'opposent les esquisses de subjectivation qui laissent entrevoir les initiatives visant à recréer du travail, les circulations, les réunions, les conflits, eux-mêmes (...). Les conflits, lorsqu'ils arrivent, sont stoppés par des retours forcés ou par des déplacements vers d'autres camps. Les activités rémunérées restent informelles, même si certaines sont tolérées, de même que les circulations hors des camps, toujours dérogatoires... La ville est bien au cour du camp, mais elle n'y est jamais que sous la forme d'ébauches perpétuellement avortées » (Agier 2008 : 218-219).

9. Ce fût le cas de la LOPPSI II. 


\section{RÉFÉRENCES}

Acovitsiòti-Hameau, A. \& Hameau, Ph. 1996 «Conversion artisanale et volonté identitaire : l'exemple des charbonniers de Moyenne Provence », Techniques \& culture 28 : 107-152.

Acovitsiòti-Hameau, A. 2000 Transformer et habiter la forêt : les sites de charbonnage dans le Var. In B. Brun, A.H. Dufour \& al. (dir.) Cabanes, cabanons et campements. Formes sociales et rapports à la nature en habitat temporaire. Châteauneuf : Éditions de Bergier : 109-121.

Agier, M. 2002 Aux Bords du monde, les réfugiés. Paris : Flammarion.

- 2008 Gérer les indésirables. Des camps de réfugiés au gouvernement humanitaire. Paris : Flammarion.

Aubin, E. 1996 « 1912-1969 La Liberté d'aller et venir : l'idéologie sécuritaire », Études Tsiganes 7 : 13-37.

Baghioni, L. 2007 Approche ethnographique des saisonniers du tourisme. Paris : Université de Paris V-Sorbonne (Mémoire de recherche).

Bauman, Z. 2004 Wasted Lives Modernity and its Outcasts. Cambridge : Polity Press Ltd.

Bernard, N. 2006 Maîtriser son logement, réflexion sur l'inadaptation des instruments législatifs, Droit et société 63-64: 553-583.

Birh, A. 2007 La précarité gît au cœur du rapport salarial. Une perspective marxiste? Interrogations? (4) : 4- 21.

Boltanski, L \& Chiapello, E. 1999 Le Nouvel esprit du capitalisme. Paris : Gallimard.

Bouillon, F. 2009 Les Mondes du squat. Paris : Presses Universitaires de France.

Bourdieu, P. 2008 [1998] Contre-feux. Paris : Raisons d'agir.

Calame, F. 1987 Peau de bois, peau de pierre. Permanence et fragilité de l'habitat, Terrain 9: 82-92.

Certeau (de), M. 1990 [1980] L'Invention du quotidien. 1 Arts de faire. Paris : Gallimard.

Charlemagne, J. 1996 - 1990 La Loi Besson : la prééminence de l'enjeu politique, Études Tsiganes 7 : 53 - 69.

Cresswell, R. 1976 Techniques et culture. Les bases d'un programme de travail, Techniques et culture Bulletin de l'équipe de recherche 191 (1) : 7-59.

Deleuze, G. \& Guattari, F. 1980 Mille plateaux. Paris : Les éditions de Minuit.

Desbois, E. 2002 Le Gourbi des saute-en-l'air, Communications 73 : 31-48.

Gaboriau, P. 1993 Clochard. L'univers d'un groupe de sans-abri parisien. Paris : Juliard.

Goffman, E. 1984 [1961] Asiles : Études sur la condition sociale des malades mentaux. Paris : Éditions de Minuit. Jeanjean, A. 2007 Waste and Powers : Political and Symbolic Effects Due to Privatization of Urban Sanitation Services, Anthropology of the Middle East 1-2 : 108-114.

Le Blanc, G. 2007 Vies ordinaires vies précaires. Paris : Seuil.

Legros, O. 2010 Les « Villages d'insertion » : un tournant dans les politiques en direction des migrants roms en région parisienne? Revue Asylon(s) 8 [en ligne] http : www.reseau-terraeu/article 947.html. (consulté le 4 juillet 2011).

Lévi-Strauss, C. 1995 [1955] Tristes tropiques. Paris : Plon.

Marx, K. \& Engels, F. 1847 Manifeste du parti communiste [en ligne] http://www.marxist.org/français/marx/ works/1847/00/kmfe18470000.htm (consulté le 6 juillet 2011).

Pétonnet, C. 1979 On est tous dans le brouillard. Paris : Galilée.

— 1982 Espaces habités. Paris : Galilée.

Pichon, P. 2002 Vivre sans domicile fixe : l'épreuve de l'habitat précaire, Communications 73 : 11-29.

Picon, B. 2000 Les cabanes de l'entre deux monde. In B. Brun, A.H. Dufour \& al. (dir.) Cabanes, cabanons et campements. Formes sociales et rapports à la nature en habitat temporaire. Châteauneuf : Éditions de Bergier : 327-334.

Poulain, F. 2005 Le Guide du camping-caravaning sur parcelles privées. Paris : Cheminements.

Raveneau, G. \& Sirost, O. 2011 Anthropologie des abris de loisirs. Paris : Presses Universitaires de Paris X.

Remy, J. 1996 Du Statut de l'interstice dans la dynamique spatiale : le pays de Herve comme cas de figure [en ligne] http//www.bsglg.be/uploads/BCGLg-1996-32-17_REMY--, pdf (consulté le 4 juillet 2011).

Trasher, F. 1927 The Gang : A Study of 1313 Gangs in Chicago. Chicago : University of Chicago Press. 


\section{RÉSUMÉ}

Des-Équilibres humains. Une introduction. Cette introduction s'appuie sur des auteurs pour la plupart absents du Thema. Il s'agit dès lors de situer ce volume dans le contexte contemporain, d'en montrer les intentions, les limites et les particularités. L'accent est mis sur les habitations occupées et construites "sans droit ni titre », les pratiques, les rythmes et les matériaux. Les rapports de norme, les dimensions politiques qui traversent les actes de construire et d'habiter sont au cœur de la réflexion.

\section{ABSTRACT}

Human Imbalances. An introduction This introduction is based on the work of authors who, for most of them, are not present in the Thema. The aim is to place this volume back into the contemporary context as well as to clarify its intentions, limits and particularities. It focuses on housing occupied and built without legal rights or documents, on practices, rhythms and materials used. Relations of norms and political dimensions, as part of the process of building and living in, are at the heart of the thinking.

\section{MOTS CLÉS}

Habitat de fortune, rapports de norme, précarité, flexibilité, mobilité, pratiques interstitielles

\section{KEYWORDS}

Shanty housing, relations of norm, precariousness, flexibility, mobility, interstitial practices 